Recibido 05 de octubre, 2017 - Aceptado 05 de noviembre, 2017

\title{
Formación en Investigación Científica y El aprendizaje de Informe de Tesis en El Instituto Superior Pedagógico Público Coracora
}

\section{Training in Scientific Research and Learning of The Thesis Report at the Upper Pedagógico Público Coracora Institute}

\author{
Efrain Taype ${ }^{1}$
}

\section{RESUMEN}

Los resultados del estudio de enfoque cuantitativo y diseño correlación confirman que entre las variables de formación en investigación científica y el aprendizaje de informe de tesis, se encuentran en una discrepancia entre promedio regular y bueno, cuyo objetivo de la investigación viene a ser determinar la relación que existe entre las variables.

Formación en investigación científica y el Aprendizaje de informe de tesis en los estudiantes del IX ciclo del Instituto Superior Pedagógico público Coracora, donde se determinó las variables estudiadas que necesitan replantear sus estrategias de aprendizaje para satisfacer a los estudiantes, en cuanto a investigación científica para elaborar un informe de tesis con los parámetros académicos establecidos, se aplicó el software estadístico SPSS versión 21, empleándose la fórmula de Rho de spearman con un margen de error al 5\%, donde se estableció la escala de frecuencias, las correlaciones, la confiablidad de los instrumentos y recolección de datos.

Palabras claves: Formación, investigación científica, aprendizaje, informe de tesis.

\section{ABSTRACT}

The results of the study of quantitative approach and correlation design confirm that between the variables of scientific research training and the learning of the thesis report, they are in a discrepancy between regular and good average, whose objective of the investigation is to determine the relationship that exists between the variables.

Training in scientific research and Learning thesis report in students of the ninth cycle of the Instituto Superior Pedagógico public Coracora, where the variables studied were determined that need to rethink their learning strategies to satisfy students, as To scientific research to develop a thesis report with the established academic parameters, SPSS statistical software version 21 was applied, using the spearman's Rho formula with a margin of error of 5\%, where the frequency scale was established, the correlations, the reliability of the instruments uments and data collection

Keywords: Training, scientific research, learning, thesis report. 


\section{INTRODUCCIÓN}

En el Perú, la investigacion cientifica se encuentra entre los países mas bajos que menos invierte en I+D en la región, apenas estamos por encima de Ecuador y Paraguay, países como Brasil y Chile invierte más que el Perú (1).

En toda institución se debe establecer la importancia de la investigación como uno de sus pilares fundamentales, ya que no todos los conocimientos se adquieren en los libros o en las exposiciones del docentes, sin embargo el proceso de la investigación cientifica puede parecer compleja si es que no se determinan las estrategias y/o métodos de aprendizaje idoneas para la institución educativa. Estos conocimientos con influencia investigativa son necesarios para que los estudiantes puedan elaborar un informe de tesis de manera organizada y sistematizada, sin embargo es una responsabilidad de las instituciones educativas plantearse esos objetivos en beneficio de las investigación cientifica.

Acudiendo a Piaget, el conocimiento se produce a través de las estructuras cognitivas, desde una observacion hasta la elaboracion semántica, donde se destaca que las universidades deben ser conscientes que la mayoría de sus egresados no harán investigación como su actividad principal, sin embargo, deben tener la capacidad de hacer investigación y realizarla cuando sea necesaria en su actividad laboral (2).

La investigación científica se debe a una disciplina constante, no deja los hechos a la casualidad, se basa en fenómenos observables que pueden deducirse o inferirse de la realidad y juzga de manera objetiva, en otras palabras, es sistemática, controlada, empírica y crítica, lo cual permite que la humanidad evolucione y progrese científica y tecnológicamente (3).

Considerando que la investigación científica se define como el proceso que permite la interacción del proceso a resolver, es el objeto a investigar (4).
Así se señala que la investigación es un proceso formal, sistemático e intensivo de llevar a cabo el método científico del análisis donde comprende una estructura de investigación más sistemática, que desemboca generalmente en una especie de reseña formal de los procedimientos y en un informe de los resultados o conclusiones (5).

En una investigación "Docencia y formación científica universitaria”, presentada en la Pontificia Universidad Javeriana Colombia. BogotáColombia, se presentó como el objetivo analizar el contexto de las comunidades académicas y las posibilidades de un desarrollo docente que incorpore el tema científico de manera significativa, en los procesos de formación y en el horizonte de la incorporación de los estudiantes a los sistemas de investigación universitaria, y la metodología que se usó en esta investigación fue de tipo descriptiva (6).

El objetivo del presente artículo es divulgar los resultados del estudio dirigido determinar el desempeño del tutor en el proceso de acompañamiento al participante del doctorado en la producción científica (7). En materia de estudio los objetivos son los siguientes:

a) Identificar la relación entre la evaluación de la formación en investigación científica con el aprendizaje de la dimensión planteamiento del estudio de tesis en los estudiantes del IX ciclo del instituto superior pedagógico público Coracora.

b)Investigar la relación entre la evaluación de la formación en investigación científica con el aprendizaje de la dimensión marco teórico de tesis en los estudiantes del IX ciclo del instituto superior pedagógico público Coracora.

c)Determinar la relación entre la evaluación de la formación en investigación científica con el estudio empírico marco teórico en los estudiantes del IX ciclo del instituto superior pedagógico público Coracora. 
d)Establecer la relación entre la evaluación de la formación en investigación científica con el aprendizaje de la dimensión bibliografía, anexos $\mathrm{y}$ aspectos preliminares en los estudiantes del IX ciclo del instituto superior pedagógico público Coracora.

e)Identificar la relación entre la evaluación de la formación en investigación científica con el aprendizaje de la dimensión redacción e informe final de tesis en los estudiantes del IX ciclo del instituto superior pedagógico público Coracora.

\section{MATERIALES Y MÉTODOS}

El tipo de investigación es de tipo correlacional no experimental en la medida que el objetivo de la investigación viene a ser determinar la relación que existe entre las variables Formación en inves- tigación científica y el Aprendizaje de informe de tesis en los estudiantes del IX ciclo del Instituto Superior Pedagógico público Coracora.

Aplicándose el 20\% de la población lo cual corresponde a la muestra de 31 estudiantes, las cuales se obtuvieron mediante un muestreo probabilístico aleatorio simple, desarrollando un enfoque cuantitativo de diseño correlacional, utilizando el software estadístico SPSS versión 21, cuya fórmula de Rho de Spearman arrojo un margen de error de 5\%, donde se estableció la escala de frecuencias, las correlaciones, la confiablidad de los instrumentos y recolección de datos.

El instrumento usado fue un cuestionario para cada variable, el cual ha sido sometido a juicio de expertos, contrastando las hipótesis.

\section{RESULTADOS}

Gráfico $\mathrm{N}^{\circ} 1$ : Porcentajes de la variable $\mathrm{x}$ : formación en investigación científica.

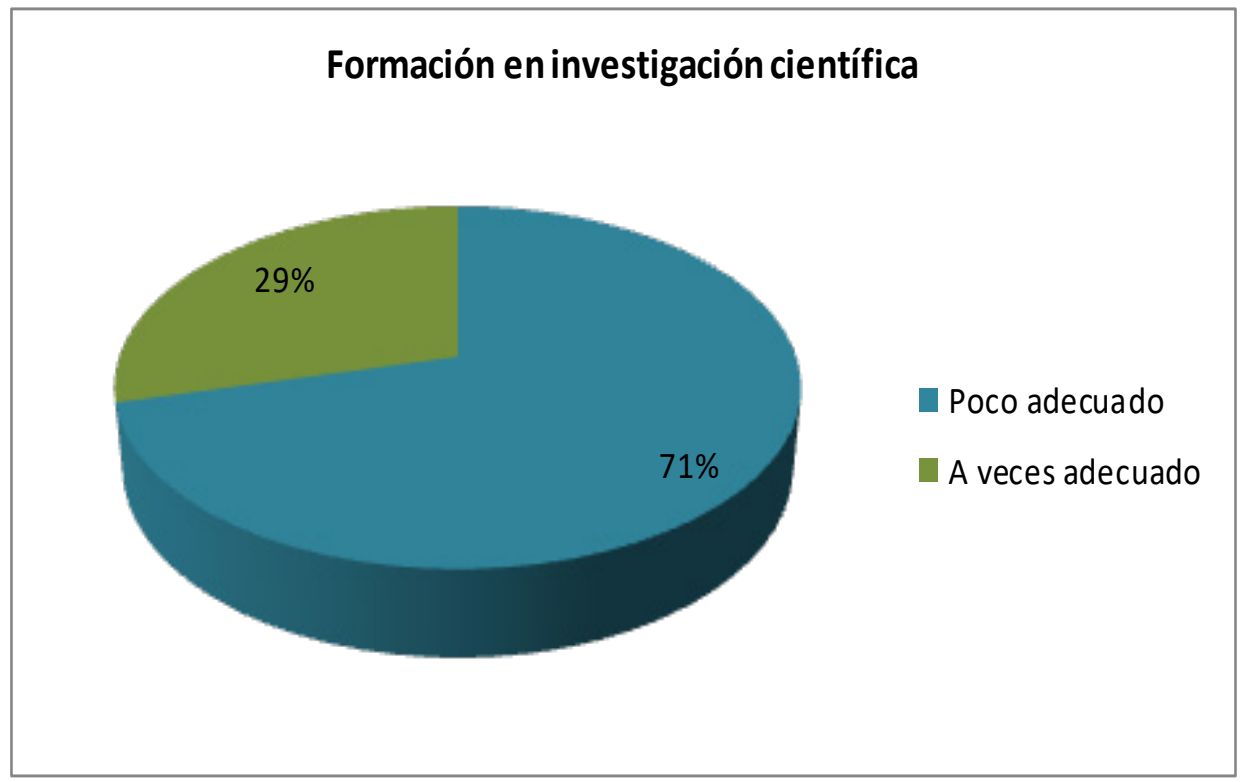

Resultado del porcentaje de formación en investigación científica. 


\section{Gráfico $N^{\circ} 2$ : Porcentajes de la variable Y: aprendizaje en la elaboración de tesis}

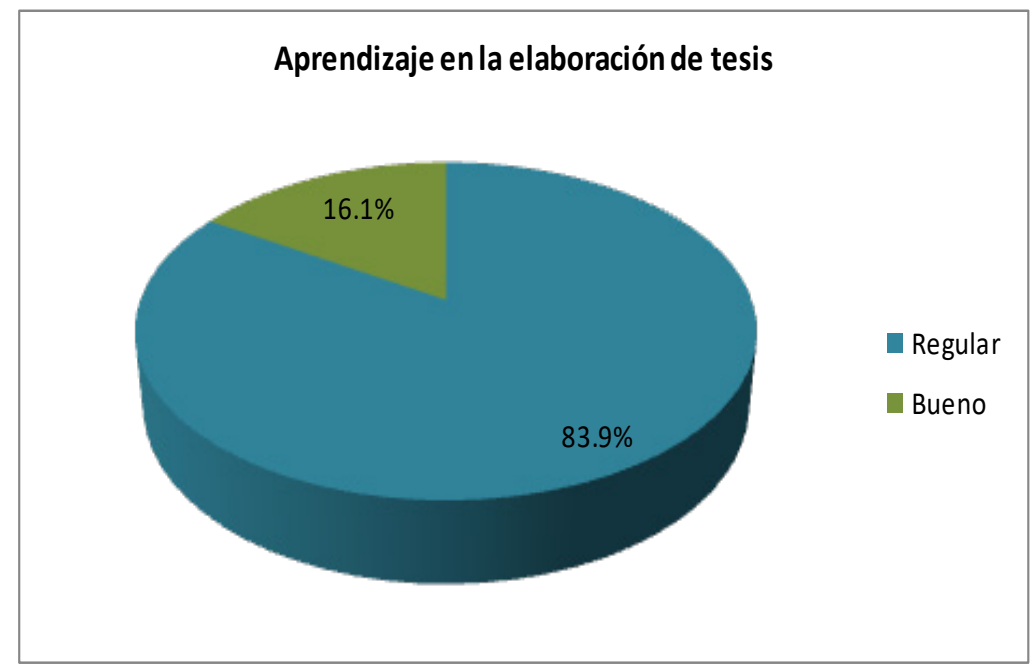

Resultado del porcentaje en aprendizaje en la elaboración de tesis.

\section{Gráfico $N^{\circ} 3$ : Porcentajes de la dimensión redacción e informe final}

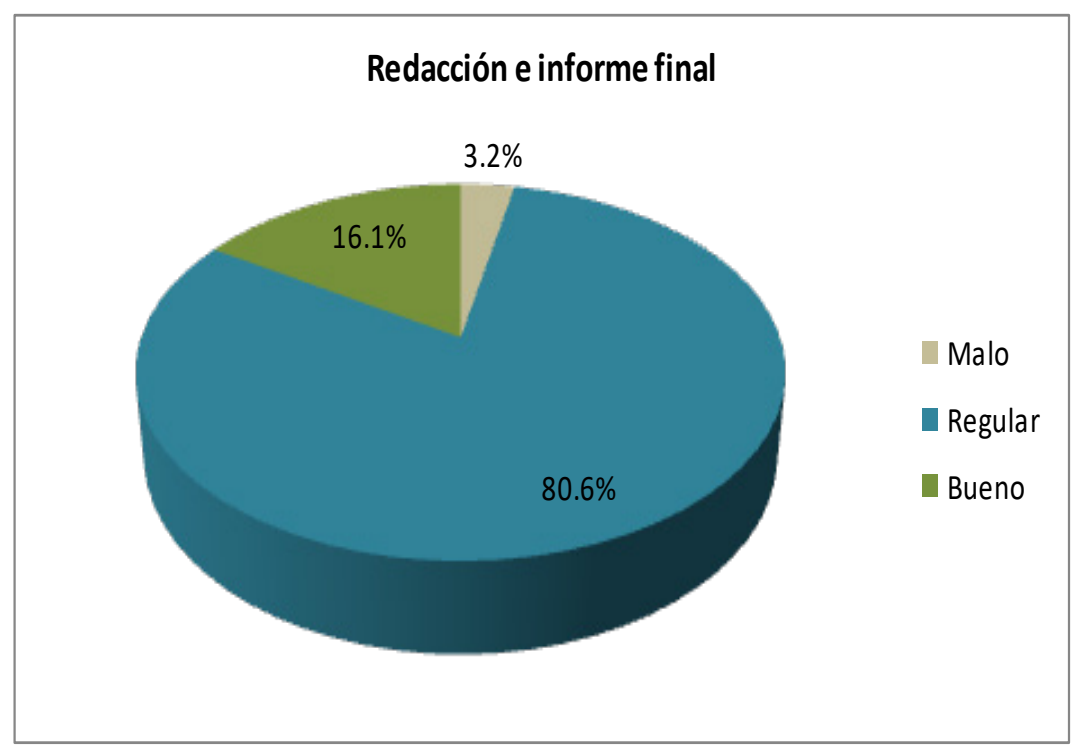

Resultado del porcentaje en redacción e informe final.

\section{DISCUSIÓN}

Los resultados de la investigación, contrastando con la hipótesis fueron estadísticamente un coeficiente de correlación buena de Rho $=0.686$, entre las variables formación en investigación científica y el aprendizaje de informe de tesis en la población estudiada.
Encontrándose respuestas divididas de parte de la muestra, representadas en la variable formación en investigación científica cuyas respuestas determinaron que el $71.0 \%$ lo considera poco adecuado frente a un $29.0 \%$ como a veces adecuado. Asimismo, para la variable aprendizaje en la elaboración de tesis las respuestas también se encuentran divididas, el $83.9 \%$ alcanzó un pro- 
medio regular, mientras que el $16.1 \%$ un promedio bueno. Con ello se manifiesta que las variables estudiadas aun no satisfacen totalmente a los miembros de la institución en estudio lo cual impide alcanzar con los objetivos académicos.

Del mismo modo, en el análisis de la primera hipótesis específica se determinó un coeficiente de correlación moderada de Rho $=.420$ entre la variable formación en investigación científica y la dimensión planteamiento del estudio de la población estudiada, encontrándose diferencias en la muestra representada en la variable de formación en investigación científica cuyas respuestas determinaron que el $71.0 \%$ lo considera poco adecuado frente a un $29.0 \%$ como a veces adecuado.

Asimismo, para la dimensión planteamiento del estudio las respuestas también se presentan diferencias, el $74.2 \%$ se encuentra en un nivel regular frente a un $12.9 \%$ en un nivel bueno, con lo cual se confirma que la variable y la dimensión analizada aún necesitan adaptar nuevas estrategias para la enseñanza del planteamiento del estudio para alcanzar los objetivos académicos con respecto a la investigación científica en la institución analizada.

El análisis de la segunda hipótesis específica, determinaron estadísticamente un coeficiente de correlación moderada de Rho $=.490$, entre la variable formación en investigación científica y la dimensión marco teórico, encontrándose respuestas divididas de parte de la muestra: variable formación en investigación científica, cuyas respuestas determinaron que el $71.0 \%$ lo considera poco adecuado frente a un $29.0 \%$ como a veces adecuado, asimismo para la dimensión marco teórico las respuestas también se encuentran divididas, ya que el $77.4 \%$ alcanzó un promedio regular y un $12.9 \%$ un promedio malo.

Con lo cual se confirma que la variable y la dimensión analizada aún necesitan adaptar nuevas estrategias para la enseñanza del marco teórico para alcanzar los objetivos académicos con res- pecto a la investigación científica en la institución analizada.

En la tercera hipótesis específica, las estadísticas determinaron un coeficiente de correlación moderada de $\mathrm{Rho}=.430$, entre la variable formación en investigación científica y la dimensión estudio empírico, encontrándose respuestas divididas de parte de la muestra: variable formación en investigación científica cuyas respuestas determinaron que el $71.0 \%$ lo considera poco adecuado frente a un $29.0 \%$ como a veces adecuado, asimismo para la dimensión estudio empírico las respuestas también se encuentran divididas, ya que el $77.4 \%$ alcanzó un promedio regular y el $16.1 \%$ con un promedio malo, por ello se determina que la variable y la dimensión analizada aún necesitan adaptar nuevas estrategias en la enseñanza del estudio empírico para alcanzar los objetivos académicos con respecto a la investigación científica en la institución analizada.

Respecto a la cuarta hipótesis específica, las estadísticas determinaron un coeficiente de correlación moderada de Rho $=.484$, entre la variable formación en investigación científica y la dimensión aspectos complementarios. Encontrándose respuestas diferencias de parte de la muestra, en cuanto a la variable formación en investigación científica las respuestas determinaron que el $71.0 \%$ lo considera poco adecuado frente a un $29.0 \%$ como a veces adecuado, asimismo para la dimensión aspectos complementarios las respuestas también encuentran diferencias, puesto que un $83.9 \%$ alcanzó un nivel regular y un 9.7\% un nivel bueno, con lo cual se confirma que la variable y la dimensión analizada aún necesitan adaptar nuevas estrategias para la enseñanza de los aspectos complementarios para alcanzar los objetivos académicos con respecto a la investigación científica en la institución analizada.

En la quinta hipótesis específica, las estadísticas determinaron un coeficiente de correlación moderada de $\mathrm{Rho}=.486$, entre la variable formación en investigación científica y la dimensión redacción e informe final, encontrándose respuestas 
diferencias de parte de la muestra, en cuanto a la variable formación en investigación científica, las respuestas determinaron que el $71.0 \%$ lo considera poco adecuado frente a un $29.0 \%$ como a veces adecuado, asimismo para la dimensión redacción e informe final las respuestas también encuentran diferencias, puesto que el $80.6 \%$ logró un promedio regular y el $16.1 \%$ un buen promedio, con lo cual se halló que la variable y la dimensión necesitan adaptar nuevas estrategias de aprendizaje con los estudiantes y la ampliación de los aprendizaje para satisfacer en su totalidad a los estudiantes, referente a la investigación científica para que así la redacción del informe final siga los parámetros académicos establecidos.

\section{CONCLUSIÓN}

Los resultados hallados confirman que entre las variables formación en investigación científica y el aprendizaje de informe de tesis, se encuentran en discrepancias entre promedio regular y bueno, determinándose así que las variables estudiadas necesitan replantear sus estrategias de aprendizaje para satisfacer en su totalidad a los estudiantes, en cuanto a investigación científica para elaborar un informe de tesis con los parámetros académicos establecidos.

La variable formación en investigación científica y la dimensión estudio empírico de la población en estudio difieren en sus resultados, entre regular y malo.

Determinando que la variable y la dimensión considerar nuevos planteamientos para las estrategias de aprendizaje para satisfacer en su totalidad a los estudiantes, en la investigación científica para elaborar un estudio empírico con los parámetros académicos establecidos en el informe de tesis.

\section{BIBLIOGRAFÍA}

1. Bermúdez, J (2015). Artículo: Investigación Científica en el Perú: Factor crítico de éxito para el desarrollo del país. España.
2. Miyahira, J. (2009). La investigación formativa y la formación para la investigación en el pregrado. Disponible en: http://www.scielo.org.pe/pdf/rmh/v20n3/v20n3e1.pdf

3. Meneses, P. (2011) Guía para la Elaboración del Informe Final de Investigación o Tesis. Disponible en: http:// universidadtamaulipeca.edu.mx/invest/investigacion/DIInforme-Final.pdf

4. Bueno, E. (2003) La investigación científica: teoría y metodología. Disponible en: http://www.postgradoune.edu. pe/documentos/LA\%20INVESTIGACI\%C3\%93N\%20 CIENT\%C3\%8DFICA.pdf

5. Rojas H. (2011). "Docencia y formación científica universitaria”. Bogotá, Colombia. Disponible en: http:// revistas.javeriana.edu.co/index.php/MAGIS/article/ view/3560/2674

6. Rojas H. (2011). "Docencia y formación científica universitaria”. Bogotá, Colombia. Disponible en: http:// revistas.javeriana.edu.co/index.php/MAGIS/article/ view/3560/2674

7. Vera \& Vera (2015). “Desempeño del tutor en el proceso de acompañamiento en la producción científica", Maracaibo- Venezuela. Disponible en: http://publicaciones.urbe. edu/index.php/telos/article/viewArticle/3832/4784 